\title{
Christoph Scheuplein
}

\section{An die Wertschöpfungskette gelegt Die finanzgetriebene Restrukturierung in der deutschen Automobilzulieferindustrie und ihr Scheitern}

\section{Einleitung}

Die Akkumulation in den meisten entwickelten Volkswirtschaften wurde vor der Weltwirtschaftskrise ab dem Herbst 2008 immer stärker durch ein finanzgetriebenes Regime geprägt (Aglietta 2000, Epstein 2005). Der wachsende Einfluss des Finanzkapitals breitete sich in der Produktion von Waren und Dienstleistungen in zwei Richtungen aus. Zum einen wurde der langfristige Gewinn als wichtigste Zielgröße eines Unternehmens durch die kurzfristigen Gewinninteressen der Eigentümer abgelöst (Lazonick/O'Sullivan 2000). Dieses Eigentümerinteresse („Shareholder Value“) wurde in vielfacher Weise in den Organisations-, Kontrollund Produktionsstrukturen der Unternehmen abgebildet (Höpner 2003, Faust/ Bahnmüller/Fisecker 2011). Zum anderen wurden Unternehmen durch das externe Engagement von Finanzinvestoren geprägt. Seit dem Ende der 1970er Jahre waren spezialisierte Kapitalbeteiligungsgesellschaften entstanden, für die eine Unternehmensbeteiligung keine reine Vermögensanlage mit eher langfristiger Ausrichtung bedeutet, sondern für die das Kaufen, Umgestalten und Verkaufen von Unternehmen zum eigentlichen Geschäftsfeld geworden ist. Üblicherweise wird dabei die Eigentumsmehrheit eines Unternehmens übernommen und die unternehmerische Kontrolle ausgeübt. In allen entwickelten Volkswirtschaften ist Private Equity inzwischen von einer vereinzelten Finanzierungsform zu einer wichtigen Alternative zur Finanzierung über die Börse geworden (Kaplan/Strőmberg 2009). Die größere Bedeutung der externen Beteiligung von Finanzinvestoren wirkt auch als Motivation für eine freiwillige Internalisierung finanzkapitalistischer Ziele seitens der Unternehmen.

In Deutschland hat sich dieser Markt für Unternehmenskontrolle erst ab dem Ende der 1990er Jahre entwickelt; im Jahr 2007 wurde ein vorläufiger Gipfelpunkt erreicht (Kaserer 2007, Jowett/Jowett 2011). Zeitweise wurden große mittelständische Unternehmen im Wochentakt übernommen und die Kapitalbeteiligungsgesellschaften dominierten den Akquisitionsmarkt, auf dem 
die traditionellen Industrieunternehmen immer weniger in der Lage schienen, die Kaufpreise aufzubringen. Entsprechend aufgeladen entwickelte sich in diesem Zeitraum die wirtschafts- und gesellschaftspolitische Debatte, wobei Private Equity einerseits als „Fitnessprogramm“ (Bunker 2005) und Hilfe für unterkapitalisierte Mittelständler (Schäfer 2007, Beise/Schmittmann 2008) und andererseits als eine schädliche Auslieferung an den Kapitalmarkt verstanden wurde (Huffschmid/Köppen/Rhode 2007). Über alle kontroversen Deutungen hinweg waren sich die Kontrahenten jedoch einig in der Diagnose, dass die Übernahmewelle die deutschen Industriestrukturen durchgreifend und nachhaltig verändern werde.

Eine Weltwirtschaftskrise später soll diese These hinterfragt werden. Private Equity zeichnet sich gegenüber anderen Anlageformen dadurch aus, dass das Kapital sich vollständig in der produktiven Konsumtion befindet und es „nicht fortzirkulieren kann“ (Marx 1893: 40), bis ein erneuter Unternehmensverkauf stattgefunden hat. Damit ist diese Finanzierungsform besonders eng an den konjunkturellen Verlauf und die technologischen und arbeitsorganisatorischen Veränderungen der jeweiligen Branche gebunden. Zwar spricht Vieles dafür, dass die Widersprüche der Akkumulation, die zu einem finanzgetriebenem Regime geführt haben, weiterhin bestehen (Sablowski 2011). Davon zu trennen sind jedoch die Wirkungen, die die finanziell motivierten Investitionen innerhalb des Unternehmenssektors erzielen. Wenn Private Equity zu Recht als Speerspitze des Finanzkapitals in der Industrie verstanden wird, dann ist es fraglich, ob es seine Optimierungsabsichten tatsächlich realisieren konnte. Hat es eine nachhaltige Restrukturierung und effiziente Unternehmensstrukturen mit herbeigeführt? Konnte Private Equity gar einen Beitrag zum jüngsten konjunkturellen Boom in Deutschland leisten?

Um dies zu beantworten, werden hier die Wirkungen von Private Equity im Rahmen einer Branche nachgezeichnet. Als Fallbeispiel dient die Automobilindustrie; diese größte deutsche Industriebranche ist im vergangenen Jahrzehnt zu einem der bevorzugten Ziele von Private Equity-Gesellschaften geworden. Da keine Endhersteller, sondern nur Zulieferer übernommen worden sind, beschränkt sich die Studie auf dieses Segment. Zeitlich setzt der Betrachtungszeitraum mit dem Beginn des Jahrzehnts ein, als die Übernahmewelle in der Branche erst langsam ihren Anfang nahm. Da auf dem Höhepunkt der Übernahmewelle deutlich mehr Transaktionen durch Private-Equity-Gesellschaften als durch die brancheneigenen Industrieunternehmen vorgenommen wurden, erhoffte man sich bzw. befürchtete man in diesem Zeitraum allgemein eine durchgreifende Veränderung der Automobilzulieferindustrie durch die Kapitalbeteiligungsgesellschaften. Insbesondere wurden den Finanzinvestoren die seit längerem anstehende Konsolidierung und eine strategische Neuausrichtung der Zulie- 
ferindustrie zugetraut (VDA/PwC 2007: 16). Während die Kontrahenten in der Private-Equity-Diskussion gemeinsam die Gestaltungsmacht der Finanzakteure betonten, wird sich im Fallbeispiel zeigen, dass Private Equity zwar eine tiefgreifende Veränderung von Wertschöpfungsstrukturen angestrebt hat. Es geriet aber mit den traditionellen Branchenunternehmen in einen Konflikt und war schließlich deren Organisations- und Unternehmensstrukturen unterlegen.

Im Folgenden werden zunächst der regulationstheoretische Rahmen der Branchenanalyse vorgestellt (2.) und dann das quantitative Engagement von Private Equity in der Beispielbranche skizziert sowie seine Investitionsstrategien vorgestellt (3). Anschließend wird das Scheitern der Finanzinvestoren in der Wirtschaftskrise aufgezeigt (4.), und es werden künftige Perspektiven von Private Equity in der Branche diskutiert (5.)

\section{Finanzmarktkapitalismus, Produktionsmodelle und Wertschöpfungsketten}

In der empirischen Untersuchung über die Folgen von Private Equity existieren bislang neben Fallstudien (Kamp/Krieger 2005) vor allem Untersuchungen, bei denen Kennzahlen zum Gewinn, Umsatz oder zu den Beschäftigten für ein Sample von Private Equity-geführten Unternehmen ausgewertet werden (vgl. als Überblick aus kritischer Sicht: Watt 2008, Wright u.a. 2009, Vitols 2009: 71-78). Dagegen fehlen Analysen auf Branchenebene, mit denen man sowohl stärker verallgemeinern, als auch die Effekte der neuen Finanzierungsform innerhalb von spezifischen Wertschöpfungsstrukturen erfassen könnte. In diesem Kontext sind auch die langfristigen Veränderungen von Unternehmens- und Marktstrukturen zu thematisieren, die erst die gesellschaftspolitische Brisanz der neuen Finanzierungsform ausmachen.

Als Analyserahmen bietet sich das regulationstheoretisch informierte Konzept der Produktionsmodelle an, das wesentlich am Beispiel der Automobilbranche entwickelt worden ist (Boyer u.a. 1998, Freyssenet/Shimizu/Volpato 2002, Freyssenet 2009). Eine unternehmensbezogene Betrachtung von Typen der Produktionsorganisation, der Arbeitsbeziehungen und des Produktangebots (das Produktionsmodell) wird hier in einen Zusammenhang mit dem makroökonomischen Koordinationsrahmen (dem nationalen Wachstumsmodus) gestellt. Der Wachstumsmodus beinhaltet den Umfang und die Formen der Investitionen, des Binnenkonsums und des Exports eines Landes und beschreibt damit den Korridor, innerhalb dessen die Unternehmen ihre Profitstrategien verfolgen (Boyer/Freyssenet 2003). Dies beinhaltet auch die institutionellen, historisch herausgebildeten Grundlagen für die Nutzung von Kapital und Arbeit eines 
Landes, so dass es die Formen der Unternehmensfinanzierung und der Unternehmenskontrolle mit einbezieht. Für den Produktionsmodelle-Ansatz wurde somit der Boom der Finanzwirtschaft, der seit den 1980er Jahren von den USA und Großbritannien ausging, schnell als ein wichtiger Einflussfaktor auf die Profitstrategien in den Ländern bzw. auf die Produktionsmodelle in den einzelnen Unternehmen thematisiert (Boyer 2000). In den Wachstumsmodi der führenden Industrieländer beobachtete man eine neue finanzielle Logik, durch die alle unternehmerischen Entscheidungen sich dem ständigen Vergleich zu allen anderen Anlageklassen auf den Finanzmärkten stellen mussten. So wurden sämtliche Unternehmensabteilungen zunehmend individuell an ihrem direkten Beitrag zur Kapitalverwertung gemessen, die Auslagerung der Montagefunktionen nahm zu und die Automobilunternehmen koppelten ihre Produktverkäufe mit eigenen Finanzdienstleistungen (Konsumentenkredit, Leasing, Versicherung). In den Analysen des Produktionsmodelle-Ansatzes wurde unter anderem auf die wachsende Eigentümerschaft von institutionellen Investoren bei den Automobilherstellern verwiesen und der Einsatz von Shareholder-Value-Strategien analysiert (Dupuy/ Lung 2002, Jürgens u.a. 2002). Dies bedeutet jedoch nicht zwingend eine Angleichung der Produktionsmodelle. Vielmehr wurden basierend auf den historischen Entwicklungspfaden erneut unterschiedliche Antworten gegeben, durch die die Heterogenität der Produktionsmodelle fortgesetzt wurde. Für die deutschen Automobilunternehmen und ihre Profitstrategien der "Qualität“ (Daimler, BMW sowie Audi und Porsche im VW-Konzern) bzw. des „Volumens und der Produktdifferenzierung" (VW, Opel, Ford) wurde ein wachsender Einfluss von Finanzakteuren und -strategien gezeigt. Dabei blieben die Auswirkungen auf die Investitionsentscheidungen begrenzt, insbesondere in den Unternehmen mit einem hohen Eigentumsanteil einer Familie (BMW, Porsche) oder des Staates (VW). Zugleich wurde die weiterhin hohe Bedeutung des nationalen Finanzsystems sowie des institutionellen Kontextes, z.B. des Berufsbildungssystems, für die Branche belegt (Kädtler/Sperling 2002). Nach dem Verkauf von Chrysler durch die Daimler AG bestätigte sich diese Tendenz (Köhler2009). Dieser Verkauf markiert mit der Übernahme von Chrysler durch die Private Equity-Gesellschaft Cerberus Capital aber auch den Bedeutungsgewinn des externen Engagements von Finanzinvestoren in der Branche. Damit stellte sich für die deutsche Automobilindustrie erneut die Frage, ob die Wertschöpfungsstrukturen durch die finanzkapitalistischen Akteure dauerhaft verändert werden.

Kennzeichnend für die Wertschöpfungsstrukturen in der Automobilindustrie ist eine hierarchische Ordnung entlang des Materialflusses. Diese Hierarchie soll hier vereinfachend mit dem „Tier“-Konzept (engl. tier = Ebene) beschrieben werden. Demnach werden auf der untersten Ebene (Tier-3) standardisierte Teile, wie z.B. Schrauben, gefertigt, auf der zweiten Ebene (Tier-2) werden Kompo- 
nenten, wie z.B. Scheinwerfer, Achsen oder Stoßdämpfer, produziert. Auf der höchsten Ebene (Tier-1) werden wiederum Module, z.B. die Sitzgruppe oder das Armaturenbrett, komplett zu den Original Equipment Manufacturer (OEM) geliefert und dort von diesen in die Fahrzeuge eingebaut. Innerhalb dieser Hierarchie an Zulieferern integriert die höhere Ebene die Produkte der niedrigeren Ebene in technischer und materieller Hinsicht. Die OEM übernehmen die Technologieentwicklung und das Design, die Endmontage und den Verkauf an den Endkunden. Sie besitzen Einfluss auf die Schlüsselstellen im Machtgefüge. Auch wenn die Lieferbeziehungen und technologischen Verflechtungen sich in der Realität erheblich komplexer gestalten und mehrere Zwischenstufen existieren, wird in der empirischen Darstellung weiter unten diese Zuordnung zu drei Ebenen genutzt, um verschiedene Kompetenzen und Machtstellungen der Unternehmen zu unterscheiden.

Zentral für die Wertschöpfungsstrukturen sind zudem der Lebenszyklus eines Automodells ( 6 bis 8 Jahre) sowie die Entwicklungsdauer eines Automodells (4-5 Jahre). Beides bedingt langfristige Liefer- und Entwicklungsbeziehungen für alle beteiligten Akteure. Im Verbund mit der oligopolistischen Marktstruktur der OEM kann somit von einer hochgradig differenzierten und über vielfältige Kooperationen und Netzwerke gestaltete Wertschöpfungskette ausgegangen werden, innerhalb der die Macht asymmetrisch zugunsten der OEM verteilt ist. Die OEM üben diese Macht über die Preisgestaltung, Zertifizierungen, Qualitätskontrolle, die Beteiligungen an der Technologieentwicklung und über vielfältige weiche Formen der Unternehmenskommunikation aus.

Dieses Muster an Integration und Kontrolle hat sich in der deutschen Automobilindustrie mit dem simultanen Wachstum von OEM und Zulieferern historisch herausgebildet. Es existiert hier in einem besonders hohen Maß und wird durch die vorherrschende Qualitätsstrategie der Hersteller verstetigt. Dabei bietet das Produktprogramm mit einem Anteil des Premium-Segments von $60 \%$ eine breite Grundlage für die Qualitätsstrategie, in der trotz allen Kostendrucks auf die Zulieferer immer wieder auch der langfristige und enge Kontakt mit einer überschaubaren Anzahl von Akteuren bedacht und gepflegt werden will.

\section{Die Aktivitäten von Private Equity 2000 bis 2011}

Die Investitions- und Desinvestitionstätigkeit von Private Equity in der deutschen Automobilzulieferindustrie wird hier anhand von Daten aus branchenspezifischen Informationsdiensten, einer Sonderauswertung von Daten des Interessenverbandes BVK, einschlägigen Unternehmensdatenbanken sowie Recherchen der Fachpresse zusammengestellt. In das Sample wurden Unternehmen einbezogen, 
die - vor oder nach der Transaktion - ihren Hauptsitz in Deutschland hatten, die mehr als 100 Mitarbeiter beschäftigten und die ihren Umsatz überwiegend mit Automotive-Produkten erzielten. Nachweisbar sein musste auch ein bestimmender Einfluss der Private Equity-Gesellschaft (PEG), wobei ein Anteil von 25 $\%$ als Untergrenze gewählt wurde. Die Datenbank wird hier vom Jahresbeginn 2000 bis einschließlich des ersten Quartals 2011 ausgewertet.

In dem genannten Zeitraum haben insgesamt 163 Unternehmensübernahmen stattgefunden. Da eine Reihe von Unternehmen mehrfach an einen Finanzinvestor veräußert worden ist (Secondary Buyout), liegt die Zahl der Unternehmen, die im gesamten Zeitraum hinweg zumindest zeitweise zum Portfolio einer PEG gehörten, bei 130. Demnach befanden sich im vergangenen Jahrzehnt rund $12 \%$ aller Zulieferunternehmen mit über 100 Beschäftigten zeitweise im Besitz von Finanzinvestoren (vgl. zur Ausgangsbasis: Ehrig 2004). Insgesamt wechselten in diesen Transaktionen Unternehmen mit einem Umsatz von gut $24 \mathrm{Mrd}$. € und mit einer Belegschaft von etwa 165.000 Beschäftigten den Eigentümer, wobei sich diese Werte jeweils auf den Zeitpunkt der ersten Übernahme beziehen. Gemessen an der Zahl der Übernahmen stellt das Jahr 2005 mit 27 Unternehmen den Höhepunkt dar. Gemessen an den Umsatz- und Beschäftigungswerten sind die Jahre 2005 und 2006 als eine gemeinsame „Welle“ an Übernahmen aufzufassen: Sie vereinigen 34 \% der Beschäftigten und 36 \% der Umsätze im gesamten Zeitraum auf sich. Nach diesem Boom markiert die Wirtschaftskrise ab 2008 einen deutlichen Einschnitt. In den Jahren 2009 und 2010 wurden nur noch 22 Transaktionen realisiert, deren Beschäftigungs- und Umsatzvolumen gering ausfiel. Es handelt sich vor allem um Unternehmen, die seit Jahrzehnten am Markt etabliert sind. Nur $5 \%$ aller Unternehmen sind nach der Wiedervereinigung entstanden.

Im Vergleich zur gesamten deutschen Automobilzulieferindustrie sind in diesem Sample durchschnittlich weniger Unternehmen mit geringen Umsätzen (bis 24 Mio. €), aber auch weniger Unternehmen in der höchsten Umsatzklasse (mehr als 1 Mrd. €) vertreten. Ein ähnliches Bild ergibt sich für die Beschäftigtengrößenklassen. Räumlich sind die übernommenen Zulieferer weit gestreut, wobei sich allerdings Schwerpunkte in typischen Zulieferregionen in Bayern, Baden-Württemberg und Nordrhein-Westfalen ausmachen lassen. Umgekehrt sind Standorte in Ostdeutschland fast gar nicht enthalten. Das Produktspektrum der Automobilzulieferindustrie wird durch die Portfolio-Unternehmen vollständig abgedeckt, wobei am stärksten die Segmente Interieur (28 \%) und Exterieur (14\%) vertreten waren. Die Bereiche Motor, Antriebstrang, Fahrwerk und Karosserie decken jeweils Anteile zwischen 10 bis 12 Prozent ab. Schwächer positioniert sind dagegen die hochtechnologischen und stark wachsenden Bereiche Elektrik/Elektronik (7 \%) sowie Ingenieurdienstleistungen (1 \%). Be- 
trachtet man die Komplexität der Produkte und Dienstleistungen, dann waren die Unternehmen zum Zeitpunkt der Übernahme zu fast zwei Dritteln auf der 3. Ebene der Zulieferpyramide positioniert. Rund $30 \%$ der Zulieferer sind als Tier-2-Zulieferer anzusehen und nur fünf Unternehmen können der ersten Ebene zugerechnet werden.

Hinter dem Übernahmeprozess in der Automobilzulieferlandschaft steht eine fast ebenso hohe Zahl an Finanzakteuren, die nach ihrer Eigentümerschaft, ihren Standorten und ihren Geschäftsstrategien und -zielen hochgradig differenziert ist. Insgesamt waren 103 PEG an den Akquisitionen beteiligt, wobei an einigen Transaktionen mehrere Investoren teilgenommen haben. Rund die Hälfte der PEG hat ihren Hauptsitz in Deutschland, dabei bilden Frankfurt und München zwei in gleicher Stärke dominierende Zentren. Ein weiteres knappes Drittel der PEG hat einen ausländischen Hauptsitz und besitzt eine Niederlassung in Deutschland. Die sonstigen Finanzinvestoren operieren ohne eine deutsche Vertretung aus dem Ausland. Insgesamt lagen die Hauptsitze der ausländischen PEG zu jeweils einem Drittel in den USA, in Großbritannien sowie im sonstigen europäischen Ausland. Nach der Gesellschafterstruktur sind vor allem die PEG mit einer direkten Herkunft aus der Finanzwirtschaft (Banken, Versicherungen oder Pensionsfonds) bedeutsam. Rund $10 \%$ der PEG haben einen öffentlichrechtlichen Hintergrund, zumeist aus dem Bereich der Landesbanken. Besonders aktiv waren sowohl einige angelsächsische Branchengrößen wie die Carlyle Group und Barclays Private Equity mit jeweils sechs Übernahmen, als auch mittelständische PEGs aus dem Heimatmarkt wie die EquiVest GmbH (8 Übernahmen), die Hannover Finanz GmbH (6), die Deutsche Beteiligungs AG (5) oder die Equita Management GmbH aus dem Besitz der Familie Harald Quandt (5).

Welche Strategien haben die Finanzinvestoren nun mit den übernommenen Unternehmen verfolgt? Hier sei zunächst die Haltedauer von PortfolioUnternehmen betrachtet, denn die Beobachtung eines kurzfristig angelegten Investitionshorizonts gehört sicherlich zu den zentralen Vorwürfen gegenüber Private Equity. Betrachtet man die Transaktionen der Jahre 2000 bis 2004 , über die bereits verlässliche Aussagen getroffen werden können, dann liegt die durchschnittliche Haltedauer bei 4 Jahren und 4 Monaten. Dieser Durchschnitt erhöht sich auf sechs Jahre, wenn man die acht Quick-Flips, d.h. den Kauf und Verkauf eines Unternehmens in weniger als zwei Jahren, heraus rechnet. Diese Fristen sind auch beachtlich, wenn man die Laufzeiten von 7 bis 10 Jahren bedenkt, auf die das Geschäftsmodell von Private Equity üblicherweise ausgelegt ist (vgl. BVK 2008: 11). Auch wenn man die Entwicklung der seit dem Jahr 2005 erworbenen Unternehmen betrachtet, bestätigt sich das Bild. Die Gründe für diese Haltedauer führen mitten hinein in die Turbulenzen zwischen Finanzkapital und industriellem Kapital in den vergangenen Jahren. Zum einen 
hat die Mehrzahl der Kapitalbeteiligungsgesellschaften nicht nur einfache Kostensenkungsprogramme oder Maßnahmen des Finanzmanagements verfolgt, sondern auch eine strategische Neuorientierung versucht. Zum anderen aber ist der Markt für Unternehmensverkäufe dieser Branche nach dem Herbst 2008 zusammengebrochen. Die lange Haltedauer vieler Unternehmen verdankt sich vor allem der Tatsache, dass der Exit eines Portfolio-Unternehmens seitdem nur mit einem Abschlag gegenüber dem ursprünglichen Kaufpreis möglich ist. Selbst wenn eine schnelle Optimierung in der Absicht vieler Finanzinvestoren gelegen haben mag, so hat sich hinter dem Rücken der Akteure der Zwang zu einer längeren Kapitalbindung geltend gemacht.

$\mathrm{Zu}$ den Maßnahmen von Private Equity zählt in vielen Fällen zunächst ein Werttransfer vom Betriebskapital, von den Beschäftigten oder von den Steuerzahlern in Richtung Finanzinvestor. Dies geschieht etwa durch eine Erhöhung des Verschuldungsgrades, durch Arbeitsplatzabbau und die Verschlechterung der Beschäftigungsbedingungen sowie durch die Reduzierung der Steuerzahlungen (Berg/Gottschalg 2003: 17-25, Kaserer u.a. 2007: 94-99 aus betriebswirtschaftlicher Sicht). Insbesondere die Überwälzung des Fremdkapitals, das zur Finanzierung des Kaufpreises aufgenommen worden war, auf die übernommenen Unternehmen kann für eine Reihe von Fällen aufgezeigt werden und destabilisierte diese Unternehmen. Auf diese operativen und finanziellen Maßnahmen können die Aktivitäten der PEG jedoch nicht reduziert werden, und dies erklärt auch nicht die besonderen Probleme in der Branche in den späteren Jahren. Eine strategische Neuausrichtung bedeutet im Kontext der Automobilzulieferindustrie, dass die Position in der oben dargestellten Wertschöpfungskette gestärkt bzw. verbessert werden muss. Dies kann z.B. durch allgemeine betriebswirtschaftliche Methoden wie die Arbeit an der Innovationskraft, an der Effizienz der Produktion oder der Internationalisierung des Unternehmens geschehen. Vor allem spielen die Private-Equity-Gesellschaften aber ihre Kernkompetenz aus: das Kaufen und Verkaufen von Unternehmen. Wachstumsorientiert und strategisch genutzt wird diese Kernkompetenz in der Buy-and-Build-Strategie (BB-S). Hier wird zunächst eine erste Unternehmensübernahme ('platform') getätigt, der weitere Käufe von Unternehmen ('add-on') folgen, wobei diese Käufe technologisch oder wirtschaftliche die Kompetenzen des ersten Unternehmens sinnvoll steigern oder ergänzen sollen. Mittelfristig sollen die Unternehmen zu einer neuen Einheit mit einem höheren Niveau der Produktionskapazität, des Produktangebots etc. integriert werden (Hoffmann 2008: 144-150).

Von allen 130 Unternehmen wurden etwa $42 \%$ in eine BB-Strategie einbezogen. In weiteren zwei Fünfteln der Unternehmen wurde ein Mix aus Maßnahmen, z.B. der internationale Ausbau von Produktionsstandorten, eine Reorganisation der Arbeitsorganisation oder der Ausbau von FuE-Kapazitäten, durchgeführt. 
Da die BB-Strategie aus forschungspraktischer Sicht leicht nachzuweisen ist und eindeutig als eine aktive Reorganisation der Wertschöpfungskette charakterisiert werden kann, konzentrieren sich die folgenden Ausführungen auf diese Strategie.

Von den 55 Unternehmen, die der BB-Strategie zugeordnet werden konnten, sind 30 als Plattform-Unternehmen (PFU) anzusehen, während 25 Unternehmen als Add-on oder als Aufbau-Unternehmen (AU) innerhalb dieser Strategie dienten. Dieses Zahlenverhältnis erscheint nur deshalb paradox, weil hier die ausländischen Unternehmen, die einem deutschen Plattform-Unternehmen angefügt worden sind, nicht als Add-on-Unternehmen gezählt werden. Tatsächlich liegt die Gesamtzahl der AU gut doppelt so hoch. Zeitlich gesehen vollzog sich der Akquisitionsprozess der Buy-and-Build-Unternehmen vor allem in den Jahren 2004 bis 2008. Entsprechend kann ein größerer Teil der Übernahmewelle in der Mitte des Jahrzehnts darüber erklärt werden, dass an einige zunächst erworbene Plattform-Unternehmen passfähige Add-on-Unternehmen angegliedert wurden.

Von den anderen übernommenen Unternehmen unterscheiden sich die Buy-and-Build-Unternehmen durch einen deutlich höheren Anteil an Tier2-Zulieferern, der vor allem auf die Plattform-Unternehmen entfiel. Die Addon-Unternehmen sind dagegen zu drei Vierteln auf der untersten Zuliefererebene angesiedelt. Tier-1-Zulieferer sind bei den Plattform-Unternehmen ebenfalls schwach, aber leicht stärker als im Gesamtbestand vertreten. Die Produktsegmente sind bei den Buy-and-Build-Unternehmen ähnlich wie im Gesamtbestand breit gestreut; ein deutlich stärkerer Schwerpunkt liegt beim Interieur-Segment, dem ein Drittel aller Unternehmen zugerechnet werden kann. Da zudem der Exterieur-Bereich überdurchschnittlich vertreten ist, kann gesagt werden, dass Buy-and-Build-Strategien vor allem in Bereichen der industriellen Massenfertigung mit einem mittleren bis einfachen Schwierigkeitsgrad der Technologie und mit einem Konsolidierungsbedarf aufgrund von Überkapazitäten angewendet wurden.

Die Buy-and-Build-Strategie orientiert sich bei den Plattform-Unternehmen auf unterschiedliche Ziele. Zu beobachten ist der Aufbau von integrierten Systemanbietern, horizontale Konzentrationsstrategien in einem Produktsegment sowie die Etablierung von spezialisierten Unternehmen auf allen Triade-Märkten. Insgesamt haben die Private-Equity-Gesellschaften mit diesem Akquisitionskurs eine aktive und strategische Rolle gespielt, die den Anspruch hatte, die Wertschöpfungsstrukturen in der Zulieferindustrie zu verändern. Es wäre aus diesem Grund ein besseres Renditeergebnis für die Buy-and-Build-Unternehmen gegenüber dem Durchschnitt der sonstigen Unternehmen zu erwarten gewesen. In der Bewährungsprobe der Weltwirtschaftskrise ergab sich jedoch ein ganz anderes Bild. 


\section{Das Scheitern in der Wirtschaftskrise}

Die im September 2008 weltweit ausgebrochene Finanzkrise schlug in nur wenigen Wochen auf die gesamte Wirtschaft durch. Dabei war die Automobilindustrie weit überdurchschnittlich betroffen und die meisten Zulieferer hatten im Jahr 2009 Umsatzrückgänge von $40 \%$ oder mehr zu verkraften. Bereits im November 2008 waren die ersten Insolvenzen von Automobilzulieferern in Deutschland zu verzeichnen. Darunter waren mit Henniges Automotive, TMD Friction, Tedrive Germany (dem deutschen Teil des früheren Ford-Zulieferers Visteon) Schwergewichte der Branche. Es folgten weitere spektakuläre Insolvenzen von Edscha, Plastal und der ae group im ersten Quartal 2009. Alle genannten Zulieferer waren im Eigentum von Finanzinvestoren. Tatsächlich ist die Insolvenzrate bei den Unternehmen mit einer PEG als Eigentümerin erheblich höher gewesen als bei Unternehmen mit anderen Eigentümern. Insbesondere bei den großen Unternehmenspleiten waren in dieser Wirtschaftskrise in der Automobilzulieferindustrie fast durchgehend Finanzinvestoren beteiligt.

Bezogen auf den gesamten Untersuchungszeitraum 2000 bis 2010 sind 43 der 130 Portfolio-Unternehmen bzw. genau ein Drittel in die Insolvenz geraten. Dabei konnten die Buy-and-Build-Unternehmen sich keineswegs positiv von den anderen Unternehmen absetzen, sondern sind sogar mit 25 Fällen bzw. 44\% erheblich häufiger in die Insolvenz gerutscht als die sonstigen Unternehmen (24\%). Der hohen Zahl der Insolvenzen stehen entsprechend wenige gelungene Exits gegenüber. Nur $12 \%$ der Buy-and-Build-Unternehmen konnten an einen industriellen Investor oder per Börsengang veräußert werden. Hier liegen die übrigen Unternehmen ebenfalls leicht besser mit $19 \%$. Aufgrund der Weltwirtschaftskrise war ein geregelter Exit zwischen Herbst 2008 und Frühjahr 2010 kaum mehr zu erreichen. Entsprechend befand sich zu Beginn des zweiten Quartals 2011 noch die Hälfte aller Unternehmen im Besitz von Finanzinves-

Tabelle 1: Exit-Typen von Portfolio-Unternehmen der Automobilzulieferindustrie in Deutschland in den Jahren 2000 bis $2011^{*}$

\begin{tabular}{lcccc}
\hline & $\begin{array}{c}\text { Plattform- } \\
\text { Unternehmen } \\
(\mathrm{n}=30)\end{array}$ & $\begin{array}{c}\text { Add-on- } \\
\text { Unternehmen } \\
(\mathrm{n}=25)\end{array}$ & $\begin{array}{c}\text { Plattform- } \\
\text { und Add-on } \\
(\mathrm{n}=55)\end{array}$ & $\begin{array}{c}\text { Übrige Unter- } \\
\text { nehmen } \\
(\mathrm{n}=75)\end{array}$ \\
\hline Industrie/IPO & 9,7 & 16,0 & 12,5 & 18,9 \\
Insolvenz & 38,7 & 52,0 & 44,6 & 24,3 \\
bislang kein Exit & 51,6 & 32,0 & 42,9 & 56,8 \\
\hline Summe & 100,0 & 100,0 & 100,0 & 100,0 \\
\hline
\end{tabular}

Quelle: Eigene Datenbank ${ }^{*} 1$. Quartal 2011 
toren. Die durchschnittliche Haltedauer der Portfolio-Unternehmen verlängert sich seitdem weiter und kann inzwischen als Indikator für eine Blockade der Exit-Kanäle interpretiert werden.

Der Investitionsprozess von Private Equity in die deutsche Automobilzulieferindustrie ist ökonomisch auch aus der Sicht der PEG wenig erfolgreich gewesen. Dies kann nur beschränkt an der Situation der gesamten Branche gelegen haben. Trotz Ertragsschwierigkeiten hatte die Zulieferindustrie im Betrachtungszeitraum bis in das Jahr 2008 Umsatz- und Beschäftigungsgewinne zu verzeichnen, und die Jahre 2010 und 2011 haben sich als Rekordjahre der Branche entpuppt. Sucht man nach Ursachen für das Scheitern, dann ist zunächst die Unternehmensauswahl zu berücksichtigen. Es überwogen Unternehmen mit einer standardisierten Produktion und mittlerer bis niedriger Technologieintensität. In diesen Bereichen strebten die PEG einen umfangreichen Turnaround bzw. eine Konsolidierung in den einzelnen Produktsegmenten an. Tatsächlich griffen die üblichen Maßnahmen der PEG im Rahmen der Automobilindustrie jedoch weniger, weil die Produktions- und Lieferstrukturen hier bereits vielfältig abgestimmt sind. Eine rein kostenorientierte Einkaufsstrategie mit der man zu einem weltweiten Sourcing übergeht, wird dadurch begrenzt, dass die OEM durch ihre Zertifizierungen und Lastenhefte die Wahl der Sub-Zulieferer bereits beschränken. Die Produktionsstandorte werden häufig durch die Just-in-TimeLogistik der OEM-Werke vorgegeben, so dass Standortverlagerungen nur in Einzelfällen möglich sind (Wallentowitz/Freialdenhoven/Olschewski 2009: 59-73). Umgekehrt müssen aber die Portfolio-Unternehmen dem Aufbau von Auslandsstandorten der OEM folgen. Noch schwieriger wird die Lage, wenn Zulieferer eine eigenständige technologische Position anstreben. Aufgrund der langen Produktlebenszyklen von Pkw existiert hier ein Konflikt mit den kürzeren Zeithorizonten der PEG. Zudem wird die Technologieentwicklung in der Automobilindustrie seit längerem in einem komplexen Kooperationsprozess vorangetrieben, den die OEM organisieren (Blöcker/Jürgens/Meißner 2009: 13-34). So stößt auch hier eine autonome Strategie auf zahlreiche Hürden des Umfelds.

Insbesondere mit der Buy-and-Build-Strategie haben die PEG jedoch diese Herausforderungen angenommen und eigene Gestaltungsziele gesteckt und verfolgt. Die 30 Plattform-Unternehmen stellten am ehesten den Anspruch einer Neuordnung der Zulieferindustrie dar. Dabei wurden mit einigen Transaktionen erhebliche Größenordnungen erreicht. Die Entlassung in die industrielle Eigenständigkeit gelang aber mit kaum einem dieser Plattform-Unternehmen. Im Juli 2007 konnte z.B. bei der SAF-Holland GmbH noch ein teilweiser Exit vollzogen werden, der aber erst im Mai 2010 mit dem vollständigen Ausstieg des Finanzinvestors abgeschlossen wurde. In der Zwischenzeit kam kein einziger erfolgreicher Exit mehr zustande, aber zehn Plattform-Unternehmen wurden zur Insolvenz 
angemeldet. Die latenten Konflikte zwischen den PEG und den OEM wurden durch die Buy-and-Build-Stragegie zu einem offenen Widerspruch entwickelt. So spielen die OEM bei der Reorganisation der Wertschöpfungskette und der Konsolidierung einzelner Produktsegmente seit Jahren eine ambivalente Rolle. Trotz ihres Interesses an der Kompetenzerweiterung und Effizienzsteigerung bei den Zulieferern sind sie äußerst bemüht, deren relative Abhängigkeit zu bewahren. Der von den PEG angestrebte Zuwachs an Marktmacht bei einzelnen Zulieferern läuft den strategischen Zielen der OEM diametral zuwider. Offenkundig wurde dieser Interessenkonflikt dann durch das Verhalten der OEM gegenüber den Zulieferern in der Wirtschaftskrise. Im Rahmen der Branchenkrise ist eine große Zahl an Zulieferern von OEM und 1-Tier-Zulieferern gestützt worden, z.B. durch die Vorauskasse bei Warenlieferungen oder die Unterstützung in Kreditverhandlungen mit den Banken. Diese Hilfestellung entfiel jedoch bei den Unternehmen im Eigentum von PEG (Pauly 2009). Selbst das Branchenfachblatt „Finance“ berichtete von einer allgemeinen Stimmungsmache durch die OEM und sieht die Finanzinvestoren auf eine ,schwarze Liste“ gesetzt (Schlumpberger 2011: 12). Dagegen sicherten die OEM nach der Insolvenz, wenn der Finanzinvestor das Portfolio-Unternehmen verlassen hatte, häufig das Fortleben des Zulieferers, z.B. indem sie eine Übernahme aus der Insolvenz durch ein industrielles Unternehmen vermittelten. Auf diesem Weg fanden größere Zulieferer wie Honsel, Plastal und Angell-Demmel einen neuen Industrieeigentümer (vgl. Klesse 2011 zu Honsel, Imbert 2011 zu Plastal und Angell-Demmel). Aufgrund derartiger Übernahmen hatten zu Beginn des zweiten Quartals 2011 von den 25 insolventen Buy-and-Build-Unternehmen 15 den Weg zurück in das industrielle Eigentum gefunden. Damit ist eine Internationalisierung der Eigentümer von zwei Dritteln der Unternehmen einhergegangen, wobei chinesische Investoren mit drei Übernahmen am stärksten vertreten waren.

\section{Zusammenfassung und Schlussfolgerungen}

Die große Übernahmewelle in der deutschen Automobilzulieferindustrie liegt sieben Jahre zurück, d.h. fast so lange wie die Laufzeit vieler Private Equity Buyout Fonds. Die damaligen Käufe hatten sowohl Hoffnungen auf eine durchgreifende Effizienzsteigerung wie auch Ängste vor einer Fremdbestimmung und vor einem unkontrollierten Abfluss von Betriebsvermögen ausgelöst. Aus heutiger Sicht kann erstens festgestellt werden, dass die Finanzierungsstruktur der übernommenen Unternehmen sich verschlechterte, weil die Kredite zum Kauf des Portfolio-Unternehmens dem gekauften Unternehmen aufgebürdet wurden. Dies ist aber nur in wenigen Fällen zur Hauptursache der Insolvenz geworden. 
Ein zweites Ergebnis ist, dass in der weit überwiegenden Mehrzahl der Fälle die Portfolio-Unternehmen von den Private-Equity-Gesellschaften lange gehalten wurden. Dies ist ein Beleg dafür, dass Strategien zur nachhaltigen Verbesserung der Wertschöpfung in den Portfolio-Unternehmen verfolgt wurden. Das Rückgrat dieser Ansätze bildete die Buy-and-Build-Strategie, die bei zwei Fünfteln aller erworbenen Unternehmen zum Einsatz kam. Mit ihnen wurde versucht, die Position der Portfolio-Unternehmen zu verbessern. Damit wollten die PEG die zentralen Wertschöpfungsstrukturen der Branche in ihrem Sinne zu beeinflussen. Tatsächlich fand diese strategische Neuorientierung jedoch in der differenzierten und hoch integrierten Wertschöpfungskette der Automobilindustrie mit ihren langfristigen Lieferantenbeziehungen, Kompetenzverteilungen und Machtverhältnissen keinen Zugang zu den entscheidenden Stellschrauben. Gerade die besonders ambitionierten Buy-and-Build-Ansätze gerieten in einen Widerspruch zu den Zielen der OEM, und dieser Konflikt wurde in der Weltwirtschaftskrise offen ausgetragen. Die OEM nutzten ihre Marktmacht dazu, einige der Private Equity-Akteure wieder aus der Branche hinaus zu drängen. Insgesamt sind die Unternehmen im Besitz von Finanzinvestoren in der Weltwirtschaftskrise von 2008 bis 2010 weit häufiger in die Insolvenz gerutscht als Unternehmen mit anderen Eigentümern. Schlaglichtartig deutlich wird dieses ökonomische Ergebnis an den fünf übernommenen Tier-1-Zulieferern: drei Unternehmen sind inzwischen weitgehend zerschlagen (Schefenacker, Honsel und Edscha), ein weiteres ist nach einem Notverkauf der Insolvenz entronnen (Peguform). Dabei sind die Unternehmen, in denen eine Buy-and-Build-Strategie angewandt worden ist, sogar noch stärker als der Durchschnitt aller Portfolio-Unternehmen von einer Insolvenz betroffen worden.

Konzeptionell kann dieses Resultat gedeutet werden als Konflikt zwischen neuartigen Elementen im nationalen Wachstumsmodus und den technisch-organisatorischen Kompromissen, die in den Produktionsmodellen der führenden deutschen Automobilunternehmen bestehen. Während die Finanzierungsform Private Equity die Wertschöpfung der übernommenen Unternehmen steigern wollte, ignorierte sie deren Einbindung in ein abgestimmtes System der Produktionsorganisation. Im Unterschied zu Interpretationen, nach denen die hohe Zahl an Insolvenzen der Portfolio-Unternehmen ausschließlich als Folge eines zu hohen Werttransfers bzw. über die Qualität der Private Equity-Manager erklärt wird, spricht der hier verfolgte Ansatz dafür, dass das Geschäftsmodell innerhalb des dicht vernetzten, systemisch integrierten Produktionsmodells der deutschen OEM seine Grenzen aufgezeigt bekommen hat. Der Grund für diesen Zielkonflikt zwischen der finanziellen Logik der Finanzinvestoren und der industriellen Logik ist allerdings sehr spezifisch. Im Rahmen anderer nationaler Profitstrategien bzw. anderer unternehmerischer Produktionsmodelle muss der 
Widerspruch nicht bzw. nicht in der geschilderten Härte auftreten. Aus dieser Perspektive ist der skizzierte Misserfolg in der Automobilzulieferindustrie weder auf andere Branchen noch auf andere Länder umstandslos zu übertragen - und erst recht nicht als Beleg für das generelle Ende des Finanzmarktkapitalismus $\mathrm{zu}$ werten.

Welche Zukunft hat angesichts dieses Zwischenfazits das Engagement von Finanzinvestoren in der Automobilzulieferindustrie? Mehr als die Hälfte aller insgesamt übernommenen Unternehmen war zu Beginn des zweiten Quartals 2011 im Besitz eines Finanzinvestors. Private Equity ist noch immer ein bedeutender Mitspieler, in vielen Fällen jedoch nur mehr unfreiwillig, da sich seit dem Herbst 2008 kein angemessener Verkaufspreis realisieren ließ. Durch diese (potenziellen) Verluste hat sich inzwischen die Neigung zu Investitionen in diese Branche abgekühlt. Zudem wird Private Equity von den Managern der Zulieferindustrie kritischer beurteilt (Frick/Krug 2010: 48). Vor allem aber dürfte Private Equity seine Rolle als Impulsgeber für eine Konsolidierung und für eine Neuordnung der Wertschöpfungskette wieder an die OEM verloren haben. Es ist nicht zu erwarten, dass in den kommenden Jahren in nennenswertem Maße eine Restrukturierung mit den wieder aufgefüllten Fonds der PEG vorangetrieben wird. Insbesondere Buy-and-Build-Strategien, durch die ganz offen eine Neupositionierung in der Zulieferpyramide angestrebt wurde, werden seltener werden. Die verbleibenden Portfolio-Unternehmen werden vermutlich in nennenswertem Maße an Automobilzulieferer aus den Schwellenländern veräußert werden. Diese neu auftretenden Marktakteure stellen zumindest zum gegenwärtigen Zeitpunkt nicht die Machtverhältnisse in der Wertschöpfungskette in Frage und dürften daher von den OEM akzeptiert werden.

\section{Literatur}

Aglietta, Michel (2000): Ein neues Akkumulationsregime. Die Regulationstheorie auf dem Prüfstand. Hamburg.

BDA (2007): Private Equity - Private Kapitalbeteiligungen stärken den Standort Deutschland. Volkswirtschaftlicher Argumentendienst, Heft 59,www.bda-online.de

Beise, Marc; Schmittmann, Stefan (Hg.) (2008): Geplündert? Gerettet? Durchgestartet? Wie sich der deutsche Mittelstand durch fremdes Eigenkapital verändert. München.

Berg, Achim; Gottschalg, Oliver (2003): Understanding Value Generation in Buyouts. INSEAD Working Paper Series, https://studies2.hec.fr/jahia/webdav/site/hec/shared/sites/buyoutresearch/acces_eleves/2003-42.pdf

Blöcker, Antje; Jürgens, Ulrich; Meißner, Heinz-Rudolf (2009): Innovationsnetzwerke und Clusterpolitik in europäischen Automobilregionen. Impulse für Beschäftigung. Münster.

Boyer, Robert (2000): Is a Finance-led Growth Regime a Viable Alternative to Fordism? In: Economy and Society, Jg. 29, H. 1: 111-145.

Boyer, Robert; Freyssenet, Michel (2003): Produktionsmodelle. Eine Typologie am Beispiel der Automobilindustrie. Berlin. 
Boyer, Robert u.a. (Hg.) (1998): Between Imitation and Innovation. The Transfer and Hybridization of Productive Models in the International Automobile Industry. Oxford und New York.

Bunker, Anthony (2005): Ausverkauf oder Fitnessprogramm für die deutsche Wirtschaft? Der Buy-out-Markt in Deutschland 2004 - Private Equity im Meinungsstreit. In: M\&A Review, Jg. 16, H. 5: 201-205.

BVK (2008): Fundraising 2007/2008 - Einschätzung der aktuellen Fundraising-Situation aus Sicht der deutschen Beteiligungsgesellschaften, www.bvk-ev.de/media/file/161.BVK_Fundraisingstudie_2007_050208_final.pdf

Dupuy, Claude; Lung, Yannick (2002): Institutional Investors and the Car Industry. Geographic focalisation and industrial strategies. In: Competition \& Change, Jg. 61, H. 1: 43-60.

Ehrig, Klaus (Hg.) (2004): Automobil-Zulieferer in Deutschland 2003/2004. Landsberg.

Epstein, Gerald A. (Hg.) (2005): Financialization and the World Economy. Cheltenham und Northampton.

Faust, Michael, Bahnmüller, Reinhard und Fisecker, Christiane (2011): Daskapitalmarktorientierte Unternehmen: Externe Erwartungen, Unternehmenspolitik, Personalwesen und Mitbestimmung. Berlin.

Freyssenet, Michel (Hg.) (2009): The Second Automobile Revolution. Trajectories of the World Carmakers in the 21st Century. Houndmills/Basingstoke/Hampshire.

Freyssenet, Michel; Shimizu, Koichi; Volpato, Giuseppe (2002) (Hg.): Globalization or regionalization of the European car industry? London und New York.

Frick, Siegfried; Krug, Volker (2010): Finanzierung, Konsolidierung, M\&A und Strategie - Zulieferer in einem sich drastisch wandelnden Umfeld. In: Verband der Automobilindustrie (Hg.): Money vs. Technology. Wie die Finanz-und Absatzkrise sowie der technologische Wandel die Zulieferwirtschaft verändern wird. Materialien zur Automobilindustrie 42. Berlin, 23-85.

Hoffmann, Nils (2008): German buyouts adopting a buy and build strategy. Key characteristics, value creation and success factors. Wiesbaden.

Höpner, Martin (2003): Wer beherrscht die Unternehmen? Shareholder Value, Managerherrschaft und Mitbestimmung in Deutschland. Schriften des Max-Planck-Instituts für Gesellschaftsforschung Köln 46. Frankfurt a. M.

Huffschmid, Jörg; Köppen, Margit; Rhode, Wolfgang (Hg.) (2007): Finanzinvestoren: Retteroder Raubritter? Neue Herausforderungen durch die internationalen Kapitalmärkte. Hamburg.

Imbert, Frank (2011): „Wir sind ein aktiver Konsolidierer“. In: Finance, Jg. 10, H. 4: 14-15.

Jowett, Paul; Jowett, Francoise (2011): Private Equity. The German Experience. Houndsmills.

Jürgens, Ulrich u.a. (2002): The Arrival of Shareholder Value in the European Auto Industry. A Case Study Comparison of Four Car Makers. In: Competition \& Change, Jg. 61, H. 1: 61-80.

Kädtler, Jürgen; Sperling, Hans Joachim (2002): The Power of Financial Markets and the Resilience of Operations. Arguments and Evidence from the German Car Industry. In: Competition \& Change, Jg. 61, H. 1: 81-94.

Kamp, Lothar; Krieger, Alexandra (2005): Die Aktivitäten von Finanzinvestoren in Deutschland. Hintergründe und Orientierungen. Hans-Böckler-Stiftung, Arbeitspapier 103. Düsseldorf.

Kaplan, Steven N.; Strőmberg, Per (2009): Leveraged Buyouts and Private Equity. In: Journal of Economic Perspectives, Jg. 23, H. 1: 121-146.

Kaserer, Christoph u.a (2007): Private Equity in Deutschland. Rahmenbedingungen, ökonomische Bedeutung und Handlungsempfehlungen. Norderstedt.

Klesse, Hans-Jürgen (2011): Zoff mit Daimler wegen Honsel. In: wiwo.de vom 9.5.2011, http:// www.wiwo.de/unternehmen-maerkte/zoff-mit-daimler-wegen-honsel-465586/

Köhler, Holm-Detlev (2009): From the Marriage in Heaven to the Divorce on Earth. The DaimlerChrysler Trajectory From a Regional Carmaker to a Global Premium Player. In: Freyssenet, 
Michel (Hg.): The Second Automobile Revolution. Trajectories of the World Carmakers in the 21st Century. Houndmills/Basingstoke/Hampshire, 309-331.

Köppen, Margit (2007): Private Equity-Fonds. Von der Investitionsfinanzierung zum Finanzinvestment. In: IG Metall Vorstand (Hg.): Wirtschaftspolitische Informationen, Heft 4: 1-10, http://www.igmetall.de/download

Lazonick, William; O’Sullivan, Mary (2000): Maximizing shareholder value: a new ideology for corporate governance. In: Economy and Society, Jg. 29, H. 1: 13-35.

Marx, Karl [1893]: Das Kapital. Zweiter Band. In: Karl Marx, Friedrich Engels: Werke, Bd. 24. Berlin 1963.

Pauly, Christoph (2009): Das große Sterben. In: Der Spiegel, Jg. 66, H. 51: 80-93.

Sablowski, Thomas (2011): Krise und Kontinuität des finanzdominierten Akkumulationsregimes. In: Zeitschrift für Wirtschaftsgeographie, Jg. 55, H. 1-2: 50-64.

Schäfer, Daniel (2007): Die Wahrheit über die Heuschrecken. Wie Finanzinvestoren die Deutschland AG umbauen. Frankfurt a. M.

Schlumpberger, Christian (2011): Überdreht. In: Finance, Jg. 10, H. 4: 8-13.

VDA/PwC (2007): Sector Report Automotive 2007. Private Equity in der deutschen Automobilzulieferindustrie. (Hektografiertes Manuskript) Frankfurt a.M.

Vitols, Sigurt (2009): Private equity: Financial engineering or solution to market failure. In: Hein, Eckhard u.a.(Hg.): Finance-Led Capitalism? Macroeconomic Effects of Changes in the Financial Sector. 2. Auflage, Marburg, 61-87.

Wallentowitz, Henning; Freialdenhoven, Arndt; Olschewski, Ingo (2009): Strategien in der Automobilindustrie. Technologietrends und Marktentwicklungen. Wiesbaden.

Watt, Andrew (2008): The impact of private equity on European companies and workers: key issues and a review of the evidence. In: Industrial Relations Journal, Jg. 39, H. 6: 548-568.

Wright, Mike; Bacon, Nicolas; Amess, Kevin (2009): The Impact of Private Equity and Buyouts on Employment, Remuneration and other HRM Practices. In: Journal of Industrial Relations, Jg. 51, H. 4, 501-515. 